\title{
Superconformal Bondi-Metzner-Sachs Algebra in Three Dimensions
}

\author{
Oscar Fuentealba $\odot,{ }^{1, *}$ Hernán A. González ${ }^{2, \dagger}$ Alfredo Pérez®${ }^{3, \$}$ David Tempo ${ }^{4,8}$ and Ricardo Troncoso $\circledast^{3, \|}$ \\ ${ }^{1}$ Université Libre de Bruxelles and International Solvay Institutes, ULB-Campus Plaine CP231, B-1050 Brussels, Belgium \\ ${ }^{2}$ Departamento de Ciencias, Facultad de Artes Liberales, Universidad Adolfo Ibáñez, Santiago 7941169, Chile \\ ${ }^{3}$ Centro de Estudios Científicos (CECS), Av. Arturo Prat 514, Valdivia 5090000, Chile \\ ${ }^{4}$ Departamento de Ciencias Matemáticas y Físicas, Facultad de Ingeniería, Universidad Católica de Temuco, Temuco 4780000, Chile
}

(Received 25 November 2020; accepted 25 January 2021; published 4 March 2021)

\begin{abstract}
The conformal extension of the $\mathrm{BMS}_{3}$ algebra is constructed. Apart from an infinite number of "superdilatations," in order to incorporate superspecial conformal transformations, the commutator of the latter with supertranslations strictly requires the presence of nonlinear terms in the remaining generators. The algebra appears to be very rigid, in the sense that its central extensions as well as the coefficients of the nonlinear terms become determined by the central charge of the Virasoro subalgebra. The wedge algebra corresponds to the conformal group in three spacetime dimensions $\mathrm{SO}(3,2)$, so that the full algebra can also be interpreted as an infinite-dimensional nonlinear extension of the $\mathrm{AdS}_{4}$ algebra with nontrivial central charges. Moreover, since the Lorentz subalgebra $[\operatorname{sl}(2, R)]$ is nonprincipally embedded within the conformal (wedge) algebra, according to the conformal weight of the generators, the conformal extension of $\mathrm{BMS}_{3}$ can be further regarded as a $W_{(2,2,2,1)}$ algebra. An explicit canonical realization of the conformal extension of $\mathrm{BMS}_{3}$ is then shown to emerge from the asymptotic structure of conformal gravity in three dimensions, endowed with a new set of boundary conditions. The supersymmetric extension is also briefly addressed.
\end{abstract}

DOI: 10.1103/PhysRevLett.126.091602

Introduction.-The symmetries of special relativity are embodied through the Poincaré algebra. Thus, extensions thereof turn out to play a relevant role in theoretical physics. Indeed, for relativistic systems with scale invariance, the algebra is generically enhanced to that of the conformal group, including special conformal transformations; see e.g., Refs. [1,2]. Conformal field theories, formulated in terms of these enhanced symmetries, have spanned a wealth of impressive results in a wide variety of contexts [3-7]. Besides, extensions of the Poincaré algebra that contain additional fermionic generators of spin $1 / 2$, known as super-Poincaré algebras, provide the building blocks for most of the supersymmetric field theories, enjoying a prominent and complementary source of exciting developments [8-14]. Another very interesting extension of the Poincaré algebra, known as the BMS algebra, emerged from the structure of asymptotically flat spacetimes at null infinity $[15,16]$, in which translations are enhanced to an infinite-dimensional ideal of "supertranslations." The BMS algebra can be further extended to admit "superrotations" [17-21] and it has recently attracted a great deal of attention due to its fascinating connections with soft theorems [22-24], the memory effect

Published by the American Physical Society under the terms of the Creative Commons Attribution 4.0 International license. Further distribution of this work must maintain attribution to the author(s) and the published article's title, journal citation, and DOI. Funded by SCOAP ${ }^{3}$.
[25], and the information paradox [26,27]. More recently, the robustness of the BMS algebra shows itself through its canonical realization either at null [28] or spatial infinity [29-31], and also near generic horizons [32].

It is then natural to wonder about the possible compatibility of these three time-honored, but wildly different extensions of the Poincaré algebra.

Conformal and supersymmetric extensions of the Poincaré algebra turn out to be perfectly compatible through the well-known superconformal algebra $[8,33]$. Nevertheless, the supersymmetric extension of the BMS algebra remains intriguing. Indeed, among the infinite number of supertranslations, only the subset of standard translations possesses a fermionic "square root" being spanned by four fermionic generators, at null [34] or spatial infinity [35]. Inequivalent extensions with an infinite number of fermionic generators have been proposed in Refs. [34,36,37], and it is still unclear whether they could be canonically realized even at the linearized level [38].

On the other hand, a conformal extension of BMS has been recently constructed in Ref. [39], which successfully accommodates "superdilatations." However, its structure is very different from that of the conformal group since standard special conformal transformations are not included. Thus, the BMS algebra seems to resist compatibility with the full conformal extension.

In the case of three-dimensional spacetimes, the conformal, supersymmetric, and BMS extensions of the Poincaré algebra are also well known. The compatibility of conformal and minimal supersymmetric extensions is also 
firmly established by the superconformal algebra $\operatorname{osp}(1 \mid 4)$ [40]. Interestingly, in contradistinction to the four-dimensional case, the $\mathrm{BMS}_{3}$ algebra $[41,42]$ is known to admit a fully fledged supersymmetric extension, in the sense that supertranslations possess suitable fermionic square roots, spanned by an infinite number of fermionic canonical generators [43]. However, as in four dimensions, a full conformal extension of $\mathrm{BMS}_{3}$ has not been hitherto reported. In fact, as it has been recently pointed out from entirely different approaches in Refs, [44-46] the $\mathrm{BMS}_{3}$ algebra can be suitably enlarged by superdilatations, but nonetheless, some difficulties in the closure of the algebra seem to preclude the inclusion of special conformal transformations.

The conformal $\mathrm{BMS}_{3}$ algebra.-Here we show that the conformal extension of the $\mathrm{BMS}_{3}$ algebra that incorporates superspecial conformal transformations is a nonlinear algebra. In particular, the commutator of supertranslations with special conformal transformations strictly requires the presence of nonlinear terms in the remaining generators, which become well defined provided that the $\mathrm{BMS}_{3}$-Weyl subalgebra is endowed with nonvanishing central extensions. This can be seen as follows.

It is simple to verify that the $\mathrm{BMS}_{3}$ algebra, spanned by superrotations $\mathcal{J}_{m}$ and supertranslations $\mathcal{P}_{m}$, once enlarged by superdilatations $\mathcal{D}_{m}$, admits only two nontrivial central charges. The centrally extended $\mathrm{BMS}_{3}$-Weyl algebra then reads

$$
\begin{aligned}
i\left\{\mathcal{J}_{m}, \mathcal{J}_{n}\right\} & =(m-n) \mathcal{J}_{m+n}+c\left(m^{2}-1\right) m \delta_{m+n, 0}, \\
i\left\{\mathcal{J}_{m}, \mathcal{P}_{n}\right\} & =(m-n) \mathcal{P}_{m+n}, \\
i\left\{\mathcal{J}_{m}, \mathcal{D}_{n}\right\} & =-n \mathcal{D}_{m+n}, \\
i\left\{\mathcal{P}_{m}, \mathcal{D}_{n}\right\} & =-i \mathcal{P}_{m+n}, \\
i\left\{\mathcal{D}_{m}, \mathcal{D}_{n}\right\} & =\tilde{c} m \delta_{m+n, 0},
\end{aligned}
$$

where $m, n \in \mathbb{Z}$. Vanishing commutators are omitted here and in the following. Note that in the presence of superdilatations, the Jacobi identity excludes the possibility of a nontrivial central charge in the commutator of $\mathcal{J}_{m}$ and $\mathcal{P}_{n}$.

The generators of superspecial conformal transformations $\mathcal{K}_{m}$ can then be incorporated provided that the superdilatations "level" $\tilde{c}$ coincides with the central charge of the Virasoro subalgebra $(\tilde{c}=c \neq 0)$, so that the remaining commutators of the full conformal $\mathrm{BMS}_{3}$ algebra are given by

$$
\begin{aligned}
i\left\{\mathcal{J}_{m}, \mathcal{K}_{n}\right\}= & (m-n) \mathcal{K}_{m+n}, \\
i\left\{\mathcal{K}_{m}, \mathcal{D}_{n}\right\}= & i \mathcal{K}_{m+n}, \\
i\left\{\mathcal{P}_{m}, \mathcal{K}_{n}\right\}= & -2(m-n) \mathcal{J}_{m+n}+(m-n) \Lambda_{m+n}^{(2)} \\
& -2 i\left(m^{2}-m n+n^{2}-1\right) \mathcal{D}_{m+n} \\
& +\Lambda_{m+n}^{(3)}-2 c\left(m^{2}-1\right) m \delta_{m+n, 0},
\end{aligned}
$$

where $\Lambda_{m}^{(s)}$ stands for nonlinear terms defined through

$$
\begin{gathered}
\Lambda_{m}^{(2)}=\frac{4}{c} \sum_{n} \mathcal{D}_{m-n} \mathcal{D}_{n}, \\
\Lambda_{m}^{(3)}=-\frac{4 i}{c} \sum_{n} \mathcal{J}_{m-n} \mathcal{D}_{n}+\frac{4 i}{c^{2}} \sum_{n, l} \mathcal{D}_{m-n-l} \mathcal{D}_{n} \mathcal{D}_{l},
\end{gathered}
$$

with (anomalous) conformal weight $s$. Indeed, with respect to the Virasoro subalgebra, the conformal weight of $\mathcal{J}_{m}, \mathcal{P}_{m}$ and $\mathcal{K}_{m}$ is given by $s=2$, while $\mathcal{D}_{m}$ has conformal weight $s=1$.

It is worth highlighting that the central extensions as well as the coefficients in front of the nonlinear terms of the conformal $\mathrm{BMS}_{3}$ algebra turn out to be entirely determined by the central charge $c$ of the Virasoro subalgebra, and in this sense, the algebra is very rigid. Indeed, the Jacobi identity imposes very stringent conditions suggesting that the nonlinear conformal extension of $\mathrm{BMS}_{3}$ is unique.

The wedge algebra reduces to that of the conformal group $\mathrm{SO}(3,2)$. It is recovered by restricting the integers that label the generators according to their conformal weight $s$ as $|m|<s$, dropping nonlinear terms [see Eqs. (A12) and (A13)].

Remarkably, the conformal $\mathrm{BMS}_{3}$ algebra can then also be interpreted as an infinite-dimensional nonlinear extension of the $\mathrm{AdS}_{4}$ algebra with nontrivial central charges. In this way, the classical theorem of algebraic cohomology that precludes nontrivial central extensions for semisimple algebras (see, e.g., Ref. [47]) clearly does not apply in this case due to the nonlinearity of the extended algebra.

Furthermore, as the Lorentz subalgebra $[s l(2, \mathbb{R})]$, spanned by $\mathcal{J}_{m}$ with $m=-1,0,1$, is nonprincipally embedded within the wedge algebra $[s o(3,2)][48]$, taking into account the conformal weight of the generators, the conformal extension of $\mathrm{BMS}_{3}$ can also be regarded as a $W_{(2,2,2,1)}$ algebra (see, e.g., Refs. [49,50]) [51].

It is also worth pointing out that, as it occurs for classical $W$ algebras, the conformal $\mathrm{BMS}_{3}$ algebra is well defined provided that the Virasoro central charge does not vanish; since otherwise, the coefficients that give support to the nonlinear terms would blow up. Nevertheless, this is not necessarily the case for the quantum algebra because these coefficients as well as the central extensions generically acquire corrections.

An explicit canonical realization of the conformal $\mathrm{BMS}_{3}$ algebra is performed in the next section, while the superconformal extension of $\mathrm{BMS}_{3}$ is briefly addressed in the final section.

Explicit realization: asymptotic structure of conformal gravity in 3D.-The aforementioned link between the conformal $\mathrm{BMS}_{3}$ and $W_{(2,2,2,1)}$ algebras naturally suggests an explicit realization in terms of a WZW model for $\mathrm{SO}(3,2)$ [50], so that the conformal $\mathrm{BMS}_{3}$ algebra could be obtained from the Kac-Moody extension of so(3,2) by virtue of a Sugawara-like construction [52]. The Kac-Moody currents could also be endowed with suitable constraints so that the conformal $\mathrm{BMS}_{3}$ algebra emerges from the Dirac brackets. Equivalently, the latter option can 
be holographically realized along the lines of Ref. [53], so that the constraints turn out to be automatically implemented through an appropriate choice of boundary conditions for a Chern-Simons theory of $\mathrm{SO}(3,2)$. This is explicitly performed in what follows.

As shown in Ref. [54], a Chern-Simons theory for $\mathrm{SO}(3,2)$, described by

$$
I_{\mathrm{CS}}[A]=\frac{k}{4 \pi} \int\left\langle A d A+\frac{2}{3} A^{3}\right\rangle,
$$

where the bracket $\langle\cdots\rangle$ stands for the invariant bilinear form in Eq. (A8), turns out to be related to conformal gravity in three dimensions [55,56], which admits an interesting class of black hole solutions [57] (see also Ref. [58]). Some choices of asymptotic conditions for conformal gravity in three dimensions have already been explored in Refs. [59-61], being such that the asymptotic symmetry algebra is given by the direct sum of a U(1) current with either $\mathrm{BMS}_{3}$ or two copies of the Virasoro algebra. Nevertheless, these choices do not accommodate the black holes in Ref. [57]. Thus, in what follows we propose a new set of boundary conditions that allows one to include them, and also provides a canonical realization of the conformal $\mathrm{BMS}_{3}$ algebra that emerges from the asymptotic symmetries.

Following Ref. [53], the radial dependence of the asymptotic form of the gauge field can be completely gauged away by virtue of a gauge choice of the form $A=h^{-1} a h+h^{-1} d h$, with $h=h(r)$, so that the components of the auxiliary connection $a=a_{t} d t+a_{\varphi} d \varphi$ depend only on time and the angular coordinate.

It is useful to express the generators of $\mathrm{SO}(3,2)$ in a basis that matches that of the wedge algebra described in the previous section, being precisely defined in Eq. (A12). Thus, the asymptotic behavior that we propose for $a_{\varphi}$ can be readily written in terms of deviations with respect to a reference configuration that go along highest weight generators [62]; i.e.,

$$
\begin{aligned}
a_{\varphi}= & J_{1}-\frac{\pi}{k}\left(\mathcal{J}-\frac{\pi}{k} \mathcal{D}^{2}\right) J_{-1}-\frac{\pi}{2 k} \mathcal{P} P_{-1} \\
& -\frac{\pi}{2 k} \mathcal{K} K_{-1}+\frac{2 \pi}{k} \mathcal{D} D_{0},
\end{aligned}
$$

where the dynamical fields $\mathcal{J}, \mathcal{P}, \mathcal{K}, \mathcal{D}$ are c-number functions that depend on $t, \varphi$. This falloff is maintained under gauge transformations $\delta a=d \Omega+[a, \Omega]$, where $\Omega=$ $\Omega\left[\epsilon_{\mathcal{J}}, \epsilon_{\mathcal{P}}, \epsilon_{\mathcal{K}}, \epsilon_{\mathcal{D}}\right]$ depends on four arbitrary functions of $t, \varphi$ $\left[\epsilon_{\mathcal{X}}=\epsilon_{\mathcal{X}}(t, \varphi)\right]$. The explicit form of $\Omega$ as well as the transformation law of the dynamical fields are given in Eqs. (A14) and (A16), respectively. According to Refs. [63,64], the asymptotic symmetries are preserved by the evolution in time by choosing the asymptotic form of $a_{t}$ to be generically given by

$$
a_{t}=\Omega\left[\mu_{\mathcal{J}}, \mu_{\mathcal{P}}, \mu_{\mathcal{K}}, \mu_{\mathcal{D}}\right],
$$

where the "chemical potentials" $\mu_{\mathcal{X}}=\mu_{\mathcal{X}}(t, \varphi)$ are assumed to be fixed at the boundary. The falloff of $a_{t}$ is then maintained by the asymptotic symmetries provided that the field equations hold in the asymptotic region, and the parameters $\epsilon_{\mathcal{X}}$ fulfill suitable differential equations of first order in time [see Eq. (A17)].

The asymptotic symmetry generators can then be obtained from different approaches $[65,66]$, and read

$\mathcal{Q}\left[\epsilon_{\mathcal{J}}, \epsilon_{\mathcal{P}}, \epsilon_{\mathcal{K}}, \epsilon_{\mathcal{D}}\right]=-\int\left(\epsilon_{\mathcal{J}} \mathcal{J}+\epsilon_{\mathcal{P}} \mathcal{P}+\epsilon_{\mathcal{K}} \mathcal{K}+\epsilon_{\mathcal{D}} \mathcal{D}\right) d \varphi$

The algebra of the conserved charges (8) can then be obtained from their Dirac brackets, or more directly from the transformation law of the fields in Eq. (A16) by virtue of $\left\{\mathcal{Q}\left[\eta_{1}\right], \mathcal{Q}\left[\eta_{2}\right]\right\}=-\delta_{\eta_{1}} \mathcal{Q}\left[\eta_{2}\right]$. It is explicitly given by

$$
\begin{aligned}
\{\mathcal{J}(\phi), \mathcal{J}(\varphi)\}= & -2 \mathcal{J}(\phi) \delta^{\prime}(\phi-\varphi)-\delta(\phi-\varphi) \mathcal{J}^{\prime}(\phi)+\frac{c}{2 \pi} \delta^{\prime \prime \prime}(\phi-\varphi), \\
\{\mathcal{J}(\phi), \mathcal{P}(\varphi)\}= & -2 \mathcal{P}(\phi) \delta^{\prime}(\phi-\varphi)-\delta(\phi-\varphi) \mathcal{P}^{\prime}(\phi), \\
\{\mathcal{J}(\phi), \mathcal{K}(\varphi)\}= & -2 \mathcal{K}(\phi) \delta^{\prime}(\phi-\varphi)-\delta(\phi-\varphi) \mathcal{K}^{\prime}(\phi), \\
\{\mathcal{J}(\phi), \mathcal{D}(\varphi)\}= & -\mathcal{D}(\phi) \delta^{\prime}(\phi-\varphi), \\
\{\mathcal{P}(\phi), \mathcal{D}(\varphi)\}= & -\mathcal{P}(\phi) \delta(\phi-\varphi), \\
\{\mathcal{K}(\phi), \mathcal{D}(\varphi)\}= & \mathcal{K}(\phi) \delta(\phi-\varphi), \\
\{\mathcal{D}(\phi), \mathcal{D}(\varphi)\}= & -\frac{c}{2 \pi} \delta^{\prime}(\phi-\varphi), \\
\{\mathcal{P}(\phi), \mathcal{K}(\varphi)\}= & 4\left(\mathcal{J}(\phi)-\Lambda^{(2)}(\phi)\right) \delta^{\prime}(\phi-\varphi)+2\left(\mathcal{J}(\phi)-\Lambda^{(2)}(\phi)+\mathcal{D}^{\prime}(\phi)\right)^{\prime} \delta(\phi-\varphi)-\frac{k}{c} \delta^{\prime \prime \prime}(\phi-\varphi) \\
& +\Lambda^{(3)}(\phi) \delta(\phi-\varphi)+6\left[\mathcal{D}(\phi) \delta^{\prime}(\phi-\varphi)\right]^{\prime} .
\end{aligned}
$$


so that once expanded in Fourier modes, $X=(1 / 2 \pi) \sum_{m} X_{m} e^{i m \varphi}$, it reduces to that in Eqs. (1) and (2), with $\tilde{c}=c=k$, provided that the zero mode of $\mathcal{J}_{n}$ is shifted as $\mathcal{J}_{0} \rightarrow \mathcal{J}_{0}-(k / 4 \pi)$.

It is worth highlighting that the central extensions of the conformal $\mathrm{BMS}_{3}$ algebra, in this context are determined by the Chern-Simons level $k$. This goes hand in hand with the fact that the conformal group $S O(3,2)$ is semisimple, and hence, it admits a unique invariant bilinear form being given by the Cartan-Killing metric [up to a normalization that can fixed as in Eq. (A8)].

As pointed out at the beginning of this section, the conformal $\mathrm{BMS}_{3}$ algebra can also be directly reproduced from the WZW model for $\mathrm{SO}(3,2)$ that is obtained once the solution of the constraints with our gauge choice $\left(a_{\phi}=g^{-1} \partial_{\phi} g\right)$ is substituted back into the Chern-Simons action (5), endowed with the boundary terms that yield the conserved charges in Eq. (8). The corresponding currents that fulfill the Kac-Moody extension of the so $(3,2)$ algebra can then be supplemented with second class constraints, being precisely implemented through requiring that $g^{-1} \partial_{\phi} g$ is given by Eq. (6), so that the algebra in Eq. (9) is recovered from the Dirac brackets.

A remarkable fact of the asymptotic behavior described above is that, since it accommodates the black holes in Ref. [57], it includes asymptotically (A)dS or flat three-dimensional spacetimes. Indeed, the precise value of the "cosmological constant" can be seen to be fixed by a suitable quotient of the chemical potentials. The structure of the generic form of the black holes that fit within our asymptotic conditions turns out to be very rich and intricate, and it can be carefully analyzed in terms of the conserved charges that span the conformal $\mathrm{BMS}_{3}$ algebra. This is left for a forthcoming work.

The superconformal $\mathrm{BMS}_{3}$ algebra.-The conformal, supersymmetric, and BMS extensions of the Poincaré algebra in three dimensions can be shown to be fully compatible. Indeed, the fermionic generators of the superconformal algebra $\operatorname{osp}(1 \mid 4)$, associated to the square roots of translations $(Q)$ and special conformal transformations $(S)$, admit infinite-dimensional extensions that we denote by $\psi_{m}^{[+]}$and $\psi_{m}^{[-]}$, corresponding to the square roots of supertranslations and superspecial conformal transformations, respectively.

The superconformal $\mathrm{BMS}_{3}$ algebra is then spanned by the set $\left(\mathcal{J}_{m}, \mathcal{P}_{m}, \mathcal{D}_{m}, \mathcal{K}_{m}, \psi_{m}^{[+]}, \psi_{m}^{[-]}\right)$, so that the commutators of the $\mathrm{BMS}_{3}$-Weyl subalgebra $\left(\mathcal{J}_{m}, \mathcal{P}_{m}, \mathcal{D}_{m}\right)$ are given by Eq. (1) with $\tilde{c}=c$; while the commutators of the generators of superspecial conformal transformations $\left(\mathcal{K}_{m}\right)$ with the remaining bosonic generators read as in Eq. (2), where the nonlinear term of conformal weight 3 in Eq. (4) acquires a quadratic shift in the fermionic generators, according to

$$
\Lambda_{m}^{(3)} \rightarrow \Lambda_{m}^{(3)}+\frac{2 i}{c} \sum_{n} \psi_{m-n}^{[-]} \psi_{n}^{[+]}
$$

The (anti-)commutators that involve fermionic generators read as

$$
\begin{aligned}
i\left\{\mathcal{J}_{m}, \psi_{n}^{[ \pm]}\right\} & =\left(\frac{m}{2}-n\right) \psi_{m+n}^{[ \pm]}, \\
i\left\{\mathcal{D}_{m}, \psi_{n}^{[ \pm]}\right\} & = \pm \frac{i}{2} \psi_{m+n}^{[ \pm]}, \\
i\left\{\mathcal{P}_{m}, \psi_{n}^{[-]}\right\}= & 2\left(\frac{m}{2}-n\right) \psi_{m+n}^{[+]}+\Lambda_{m+n}^{[+](5 / 2)}, \\
i\left\{\mathcal{K}_{m}, \psi_{n}^{[+]}\right\}= & -2\left(\frac{m}{2}-n\right) \psi_{m+n}^{[-]}-\Lambda_{m+n}^{[-](5 / 2)}, \\
i\left\{\psi_{m}^{[+]}, \psi_{n}^{[+]}\right\}= & \mathcal{P}_{m+n}, \\
i\left\{\psi_{m}^{[-]}, \psi_{n}^{[-]}\right\}= & -\mathcal{K}_{m+n}, \\
i\left\{\psi_{m}^{[+]}, \psi_{n}^{[-]}\right\}= & \mathcal{J}_{m+n}-i(m-n) \mathcal{D}_{m+n} \\
& -\frac{1}{4} \Lambda_{m+n}^{(2)}+2 c\left(m^{2}-\frac{1}{4}\right) \delta_{m+n, 0},
\end{aligned}
$$

where $\Lambda_{m}^{[ \pm](5 / 2)}= \pm(2 i / c) \sum_{n} \mathcal{D}_{m-n} \psi_{n}^{[ \pm]}$, and the brackets between fermionic generators are symmetric. The fermionic generators are labeled by integers or half-integers for fermionic parameters with periodic or antiperiodic boundary conditions, respectively.

Note that the conformal weight of the fermionic generators $\psi_{m}^{[ \pm]}$is given by $s=3 / 2$. For antiperiodic boundary conditions, the wedge algebra reduces to $\operatorname{osp}(1 \mid 4)$, being recovered once nonlinear terms are dropped and the labels of the generators are restricted according to $|m|<s$, where $s$ is their conformal weight. Therefore, the conformal weight of the generators of the superconformal $\mathrm{BMS}_{3}$ algebra, naturally suggests that it could be regarded as a $W_{\left(2,2,2, \frac{3}{2}, \frac{3}{2}, 1\right)}$ algebra.

As in the bosonic case, the superalgebra also appears to be very rigid, in the sense that the coefficients that characterize the nonlinear terms and all of the central extensions become completely determined by the central charge of the Virasoro subalgebra.

It is also worth pointing out that the superconformal extension of the $\mathrm{BMS}_{3}$ algebra can be interpreted as an infinite-dimensional centrally extended nonlinear extension of the super $\mathrm{AdS}_{4}$ algebra $[\operatorname{osp}(1 \mid 4)]$, suggesting the possibility of a different version of the $\mathrm{AdS}_{4} / \mathrm{CFT}_{3}$ correspondence [67], presumably topological and with enhanced symmetries.

A canonical realization of the superconformal $\mathrm{BMS}_{3}$ algebra can also be seen to arise from the asymptotic structure of conformal supergravity in three dimensions $[68,69]$, by virtue of a suitable supersymmetric extension of the new boundary conditions described by Eqs. (6) and (7) 
(work in progress). Our results could also be regarded as a (super)conformal completion of flat-space chiral (super) gravity [70,71].

As a final remark, it might be interesting to explore whether the super $\mathrm{BMS}_{3}$ algebras with $\mathcal{N}>1$ in Refs. [72-78], as well as the bosonic and fermionic higher spin extensions of $\mathrm{BMS}_{3}$ in Refs. [79-82] and [83,84], respectively, could also be compatible with the conformal extension developed here. The compatibility with other possible extensions of $\mathrm{BMS}_{3}$ as in, e.g., Refs. $[85,86]$ also deserves attention.

We thank Marcela Cárdenas, Ricardo Caroca, Joaquim Gomis, Daniel Grumiller, Marc Henneaux, Javier Matulich, Fábio Novaes, Miguel Pino, and Pablo Rodríguez for useful discussions. O. F. holds a "Marina Solvay" fellowship. This work was partially supported by the ERC Advanced Grant "High-Spin-Grav," by FNRS-Belgium (conventions FRFC PDRT.1025.14 and IISN 4.4503.15). This research has been partially supported by FONDECYT Grants No. 1171162, No. 1181031, No. 1181496, and No. 11190427. The Centro de Estudios Científicos (CECs) is funded by the Chilean Government through the Centers of Excellence Base Financing Program of Conicyt.

Appendix: Remarks on so $(3,2)$ and the conformal $\mathrm{BMS}_{3}$ algebra.-The so $(3,2)$ algebra, spanned by generators $J_{A B}$, which reads

$\left[J_{A B}, J_{C D}\right]=\eta_{A C} J_{B D}-\eta_{B C} J_{A D}+\eta_{A D} J_{C B}-\eta_{B D} J_{C A}$,

is well known to be isomorphic to the conformal algebra in three dimensions. Indeed, choosing $\eta_{A B}=\operatorname{diag}(-1,1,1,1,-1)$ and splitting the index $A$ according to $A=\{a, 3,4\}$, the following change of basis

$$
\begin{gathered}
J_{a}=\frac{1}{2} \epsilon_{a b c} J^{b c}, \quad P_{a}=J_{a 3}-J_{a 4}, \\
K_{a}=J_{a 3}+J_{a 4}, \quad D=J_{34},
\end{gathered}
$$

makes the algebra in Eq. (A1) to read as

$$
\begin{array}{r}
{\left[J_{a}, J_{b}\right]=\epsilon_{a b c} J^{c}, \quad\left[P_{a}, J_{b}\right]=\epsilon_{a b c} P^{c},} \\
{\left[K_{a}, J_{b}\right]=\epsilon_{a b c} K^{c}, \quad\left[P_{a}, D\right]=P_{a},} \\
{\left[K_{a}, D\right]=-K_{a}, \quad\left[P_{a}, K_{b}\right]=-2 \epsilon_{a b c} J^{c}+2 \eta_{a b} D .}
\end{array}
$$

Therefore, if the Cartan-Killing metric is normalized according to

$$
\left\langle J^{A B}, J_{C D}\right\rangle=-\delta_{C D}^{A B},
$$

in the "conformal basis" the nonvanishing components of the invariant bilinear metric are given by

$$
\left\langle J_{a}, J_{b}\right\rangle=\eta_{a b} ; \quad\left\langle P_{a}, K_{b}\right\rangle=-2 \eta_{a b} ; \quad\langle D, D\rangle=1 .
$$

Besides, $\operatorname{so}(3,2)$ also corresponds to the wedge algebra of the conformal extension of $\mathrm{BMS}_{3}$. In order to see that explicitly, it is useful to choose the Minkowski metric $\eta_{a b}$ in light-cone coordinates, so that its nonvanishing components read $\eta_{01}=\eta_{10}=\eta_{22}=1$, and an orientation for which the Levi-Civita symbol fulfills $\epsilon_{012}=1$. The suitable change of basis can then be defined as

$$
\begin{aligned}
J_{0} & \rightarrow-\frac{1}{2} J_{-1} ; & J_{2} & \rightarrow J_{0}, \\
P_{0} & \rightarrow-\frac{1}{2} P_{-1} ; & P_{2} & \rightarrow P_{0}, \\
K_{0} & \rightarrow-\frac{1}{2} K_{-1} ; & K_{2} & \rightarrow K_{0},
\end{aligned}
$$

and hence, the so(3,2) algebra in the new basis spanned by $\left(J_{m}, P_{n}, K_{m}, D\right)$, with $m, n=-1,0,1$, reduces to

$$
\begin{aligned}
{\left[J_{m}, J_{n}\right] } & =(m-n) J_{m+n}, \\
{\left[J_{m}, P_{n}\right] } & =(m-n) P_{m+n}, \\
{\left[J_{m}, K_{n}\right] } & =(m-n) K_{m+n}, \\
{\left[P_{m}, D\right] } & =P_{m}, \\
{\left[K_{m}, D\right] } & =-K_{m}, \\
{\left[P_{m}, K_{n}\right] } & =-2(m-n) J_{m+n}-2\left(m^{2}-m n+n^{2}-1\right) D,
\end{aligned}
$$

which agrees with the wedge algebra of the conformal $\mathrm{BMS}_{3}$ algebra provided that $i\{,\} \rightarrow[$,$] , and$

$\mathcal{J}_{m} \rightarrow J_{m}, \quad \mathcal{P}_{m} \rightarrow P_{m}, \quad \mathcal{K}_{m} \rightarrow K_{m}, \quad i \mathcal{D}_{0} \rightarrow D$.

In the basis (A12), the so(3,2)-valued parameter $\Omega$ that preserves the asymptotic form of the gauge field $a_{\varphi}$ in Eq. (6) is given by

$$
\begin{aligned}
\Omega\left[\epsilon_{\mathcal{J}}, \epsilon_{\mathcal{P}}, \epsilon_{\mathcal{K}}, \epsilon_{\mathcal{D}}\right]= & \epsilon_{\mathcal{J}} J_{1}-\epsilon_{\mathcal{K}} P_{1}-\epsilon_{\mathcal{P}} K_{1}+\left(\epsilon_{\mathcal{D}}+\frac{2 \pi}{k} \mathcal{D} \epsilon_{\mathcal{J}}\right) D \\
& +\eta\left[\epsilon_{\mathcal{J}}, \epsilon_{\mathcal{P}}, \epsilon_{\mathcal{K}}, \epsilon_{\mathcal{D}}\right],
\end{aligned}
$$

with 


$$
\begin{aligned}
\eta\left[\epsilon_{\mathcal{J}}, \epsilon_{\mathcal{P}}, \epsilon_{\mathcal{K}}, \epsilon_{\mathcal{D}}\right]= & -\epsilon_{\mathcal{J}}^{\prime} J_{0}+\left(\epsilon_{\mathcal{K}}^{\prime}-\frac{2 \pi}{k} \mathcal{D} \epsilon_{\mathcal{K}}\right) P_{0}+\left(\epsilon_{\mathcal{P}}^{\prime}-\frac{2 \pi}{k} \mathcal{D} \epsilon_{\mathcal{P}}\right) K_{0}-\frac{\pi}{k}\left[\left(\mathcal{J}-\frac{\pi}{k} \mathcal{D}^{2}\right) \epsilon_{\mathcal{J}}+\mathcal{K} \epsilon_{\mathcal{K}}+\mathcal{P} \epsilon_{\mathcal{P}}-\frac{k}{2 \pi} \epsilon_{\mathcal{J}}^{\prime \prime}\right] J_{-1} \\
& +\frac{\pi}{k}\left[\left(\mathcal{J}-\frac{3 \pi}{k} \mathcal{D}^{2}+\mathcal{D}^{\prime}\right) \epsilon_{\mathcal{K}}+2 \mathcal{D} \epsilon_{\mathcal{K}}^{\prime}-\frac{1}{2} \mathcal{P} \epsilon_{\mathcal{J}}-\frac{k}{2 \pi} \epsilon_{\mathcal{K}}^{\prime \prime}\right] P_{-1}+\frac{\pi}{k}\left[\left(\mathcal{J}-\frac{3 \pi}{k} \mathcal{D}^{2}-\mathcal{D}^{\prime}\right) \epsilon_{\mathcal{P}}-2 \mathcal{D} \epsilon_{\mathcal{P}}^{\prime}\right. \\
& \left.-\frac{1}{2} \mathcal{K}_{\mathcal{J}}-\frac{k}{2 \pi} \epsilon_{\mathcal{P}}^{\prime \prime}\right] K_{-1}
\end{aligned}
$$

so that the transformation law of the dynamical fields reads

$$
\begin{aligned}
\delta \mathcal{J}= & 2 \mathcal{J} \epsilon_{\mathcal{J}}^{\prime}+\mathcal{J}^{\prime} \epsilon_{\mathcal{J}}-\frac{k}{2 \pi} \epsilon_{\mathcal{J}}^{\prime \prime \prime}+2 \mathcal{P} \epsilon_{\mathcal{P}}^{\prime}+\mathcal{P}^{\prime} \epsilon_{\mathcal{P}}+2 \mathcal{K} \epsilon_{\mathcal{K}}^{\prime}+\mathcal{K}^{\prime} \epsilon_{\mathcal{K}}+\mathcal{D} \epsilon_{\mathcal{D}}^{\prime}, \\
\delta \mathcal{P}= & 2 \mathcal{P} \epsilon_{\mathcal{J}}^{\prime}+\mathcal{P}^{\prime} \epsilon_{\mathcal{J}}-4\left(\mathcal{J}-\frac{4 \pi}{k} \mathcal{D}^{2}\right) \epsilon_{\mathcal{K}}^{\prime}-2\left(\mathcal{J}-\frac{4 \pi}{k} \mathcal{D}^{2}\right)^{\prime} \epsilon_{\mathcal{K}}+\frac{k}{\pi} \epsilon_{\mathcal{K}}^{\prime \prime \prime}-2\left[\mathcal{D}^{\prime \prime}-\frac{4 \pi}{k}\left(\mathcal{J}-\frac{2 \pi}{k} \mathcal{D}^{2}\right) \mathcal{D}\right] \epsilon_{\mathcal{K}} \\
& -6\left(\mathcal{D} \epsilon_{\mathcal{K}}^{\prime}\right)^{\prime}+\mathcal{P} \epsilon_{\mathcal{D}} \\
\delta \mathcal{K}= & 2 \mathcal{K} \epsilon_{\mathcal{J}}^{\prime}+\mathcal{K}^{\prime} \epsilon_{\mathcal{J}}-4\left(\mathcal{J}-\frac{4 \pi}{k} \mathcal{D}^{2}\right) \epsilon_{\mathcal{P}}^{\prime}-2\left(\mathcal{J}-\frac{4 \pi}{k} \mathcal{D}^{2}\right)^{\prime} \epsilon_{\mathcal{P}}+\frac{k}{\pi} \epsilon_{\mathcal{P}}^{\prime \prime \prime} \\
& +2\left[\mathcal{D}^{\prime \prime}-\frac{4 \pi}{k}\left(\mathcal{J}-\frac{2 \pi}{k} \mathcal{D}^{2}\right) \mathcal{D}\right] \epsilon_{\mathcal{P}}+6\left(\mathcal{D} \epsilon_{\mathcal{P}}^{\prime}\right)^{\prime}-\mathcal{K} \epsilon_{\mathcal{D}} \\
\delta \mathcal{D}= & \mathcal{D} \epsilon_{\mathcal{J}}^{\prime}+\mathcal{D}^{\prime} \epsilon_{\mathcal{J}}-\mathcal{P} \epsilon_{\mathcal{P}}+\mathcal{K} \epsilon_{\mathcal{K}}+\frac{k}{2 \pi} \epsilon_{\mathcal{D}}^{\prime} .
\end{aligned}
$$

As pointed out in Ref. [63], the asymptotic form of the field equations can be obtained from the fact that the evolution in time corresponds to a gauge transformation spanned by $\Omega=\Omega\left[\mu_{\mathcal{J}}, \mu_{\mathcal{P}}, \mu_{\mathcal{K}}, \mu_{\mathcal{D}}\right]$, where $\mu_{\mathcal{X}}$ stands for the chemical potentials.

Finally, in order to maintain the falloff of $a_{t}$, the parameters $\epsilon_{\mathcal{X}}$ have to fulfill the following differential equations

$$
\begin{aligned}
\dot{\epsilon}_{\mathcal{J}}= & \mu_{\mathcal{J}} \epsilon_{\mathcal{J}}^{\prime}-\epsilon_{\mathcal{J}} \mu_{\mathcal{J}}^{\prime}+2\left(\epsilon_{\mathcal{P}} \mu_{\mathcal{K}}^{\prime}-\mu_{\mathcal{K}} \epsilon_{\mathcal{P}}^{\prime}-\frac{4 \pi}{k} \mathcal{D} \mu_{\mathcal{K}} \epsilon_{\mathcal{P}}\right)+2\left(\epsilon_{\mathcal{K}} \mu_{\mathcal{P}}^{\prime}-\mu_{\mathcal{P}} \epsilon_{\mathcal{K}}^{\prime}+\frac{4 \pi}{k} \mathcal{D} \mu_{\mathcal{P}} \epsilon_{\mathcal{K}}\right), \\
\dot{\epsilon}_{\mathcal{P}}= & \mu_{\mathcal{P}} \epsilon_{\mathcal{J}}^{\prime}-\epsilon_{\mathcal{J}} \mu_{\mathcal{P}}^{\prime}-\left[\left(\mu_{\mathcal{D}}+\mu_{\mathcal{J}}^{\prime}\right) \epsilon_{\mathcal{P}}-\mu_{\mathcal{J}} \epsilon_{\mathcal{P}}^{\prime}\right]+\mu_{\mathcal{P}} \epsilon_{\mathcal{D}} \\
\dot{\epsilon}_{\mathcal{K}}= & \mu_{\mathcal{K}} \epsilon_{\mathcal{J}}^{\prime}-\epsilon_{\mathcal{J}} \mu_{\mathcal{K}}^{\prime}+\left[\left(\mu_{\mathcal{D}}-\mu_{\mathcal{J}}^{\prime}\right) \epsilon_{\mathcal{K}}+\mu_{\mathcal{J}} \epsilon_{\mathcal{K}}^{\prime}\right]-\mu_{\mathcal{K}} \epsilon_{\mathcal{D}}, \\
\dot{\epsilon}_{\mathcal{D}}= & -\epsilon_{\mathcal{J}} \mu_{\mathcal{D}}^{\prime}-2\left(\mu_{\mathcal{K}}^{\prime}-\frac{8 \pi}{k} \mathcal{D} \mu_{\mathcal{K}}\right) \epsilon_{\mathcal{P}}^{\prime}+2 \mu_{\mathcal{K}} \epsilon_{\mathcal{P}}^{\prime \prime}+2\left[\mu_{\mathcal{K}}^{\prime \prime}-\frac{4 \pi}{k}\left(\mathcal{J} \mu_{\mathcal{K}}+2 \mathcal{D} \mu_{\mathcal{K}}^{\prime}-\frac{6 \pi}{k} \mathcal{D}^{2} \mu_{\mathcal{K}}\right)\right] \epsilon_{\mathcal{P}} \\
& -2\left[\mu_{\mathcal{P}}^{\prime \prime}-\frac{4 \pi}{k}\left(\mathcal{J} \mu_{\mathcal{P}}-2 \mathcal{D} \mu_{\mathcal{P}}^{\prime}-\frac{6 \pi}{k} \mathcal{D}^{2} \mu_{\mathcal{P}}\right)\right] \epsilon_{\mathcal{K}}+2\left(\mu_{\mathcal{P}}^{\prime}+\frac{8 \pi}{k} \mathcal{D} \mu_{\mathcal{P}}\right) \epsilon_{\mathcal{K}}^{\prime}-2 \mu_{\mathcal{P}} \epsilon_{\mathcal{K}}^{\prime \prime}+\mu_{\mathcal{J}} \epsilon_{\mathcal{D}}^{\prime} .
\end{aligned}
$$

*ofuentea@ulb.ac.be

†hernan.gonzalez@uai.cl

‡aperez@cecs.cl

§jtempo@uct.cl

troncoso@cecs.cl

[1] J. Polchinski, Scale and conformal invariance in quantum field theory, Nucl. Phys. B303, 226 (1988).

[2] Y. Nakayama, Scale invariance vs conformal invariance, Phys. Rep. 569, 1 (2015).
[3] P. Di Francesco, P. Mathieu, and D. Senechal, Conformal Field Theory (Springer, New York, 1997).

[4] M. B. Green, J. H. Schwarz, and E. Witten, Superstring Theory Vol. 1: 25th Anniversary Edition (Cambridge University Press, Cambridge, England, 2012).

[5] J.L. Cardy, Scaling and Renormalization in Statistical Physics (Cambridge University Press, Cambridge, England, 1996).

[6] C. Itzykson, H. Saleur, and J. B. Zuber, Conformal Invariance and Applications to Statistical Mechanics (World Scientific, Singapore, 1988), p. 979.

[7] R. Blumenhagen and E. Plauschinn, Introduction to Conformal Field Theory: With Applications to String Theory, 
Lecture Notes in Physics, Vol. 779 (Springer, Berlin, Heidelberg, 2009), pp. 1-256.

[8] S. J. Gates, M. T. Grisaru, M. Rocek, and W. Siegel, Superspace Or one thousand and one lessons in supersymmetry, Front. Phys. 58, 1 (1983).

[9] H. P. Nilles, Supersymmetry, supergravity and particle physics, Phys. Rep. 110, 1 (1984).

[10] H. E. Haber and G. L. Kane, The search for supersymmetry: Probing physics beyond the standard model, Phys. Rep. 117, 75 (1985).

[11] P. C. West, Introduction to Supersymmetry and Supergravity (World Scientific, Singapore, 1990), p. 425.

[12] S. P. Martin, A Supersymmetry Primer, Advanced Series on Directions in High Energy Physics, Vol. 21 (World Scientific, Singapore, 2010), pp. 1-153.

[13] S. Weinberg, The Quantum Theory of Fields. Vol. 3: Supersymmetry (Cambridge University Press, Cambridge, England, 2005).

[14] P. Binetruy, Supersymmetry: Theory, Experiment and Cosmology (Oxford University Press, Oxford, 2006), p. 520.

[15] R. Sachs, Asymptotic symmetries in gravitational theory, Phys. Rev. 128, 2851 (1962).

[16] H. Bondi, M. G. J. van der Burg, and A. W. K. Metzner, Gravitational waves in general relativity. 7. Waves from axisymmetric isolated systems, Proc. R. Soc. A 269, 21 (1962).

[17] G. Barnich and C. Troessaert, Symmetries of Asymptotically Flat 4 Dimensional Spacetimes at Null Infinity Revisited, Phys. Rev. Lett. 105, 111103 (2010).

[18] G. Barnich and C. Troessaert, Aspects of the BMS/CFT correspondence, J. High Energy Phys. 05 (2010) 062.

[19] G. Barnich and C. Troessaert, Supertranslations call for superrotations, Proc. Sci., CNCFG2010 (2010) 010 [arXiv:1102.4632].

[20] M. Campiglia and A. Laddha, Asymptotic symmetries and subleading soft graviton theorem, Phys. Rev. D 90, 124028 (2014).

[21] M. Campiglia and A. Laddha, New symmetries for the gravitational S-matrix, J. High Energy Phys. 04 (2015) 076.

[22] F. E. Low, Scattering of light of very low frequency by systems of spin 1/2, Phys. Rev. 96, 1428 (1954).

[23] S. Weinberg, Infrared photons and gravitons, Phys. Rev. 140, B516 (1965).

[24] T. He, V. Lysov, P. Mitra, and A. Strominger, BMS supertranslations and Weinberg's soft graviton theorem, J. High Energy Phys. 05 (2015) 151.

[25] A. Strominger and A. Zhiboedov, Gravitational memory, BMS supertranslations and soft theorems, J. High Energy Phys. 01 (2016) 086.

[26] S. W. Hawking, M. J. Perry, and A. Strominger, Soft Hair on Black Holes, Phys. Rev. Lett. 116, 231301 (2016).

[27] S. W. Hawking, M. J. Perry, and A. Strominger, Superrotation charge and supertranslation hair on black holes, J. High Energy Phys. 05 (2017) 161.

[28] C. Bunster, A. Gomberof, and A. Pérez, Regge-Teitelboim analysis of the symmetries of electromagnetic and gravitational fields on asymptotically null spacelike surfaces, arXiv:1805.03728.
[29] M. Henneaux and C. Troessaert, BMS group at spatial infinity: The Hamiltonian (ADM) approach, J. High Energy Phys. 03 (2018) 147.

[30] M. Henneaux and C. Troessaert, The asymptotic structure of gravity at spatial infinity in four spacetime dimensions, arXiv:1904.04495.

[31] O. Fuentealba, M. Henneaux, S. Majumdar, J. Matulich, and C. Troessaert, Asymptotic structure of the Pauli-Fierz theory in four spacetime dimensions, Classical Quantum Gravity 37, 235011 (2020).

[32] D. Grumiller, A. Pérez, M. M. Sheikh-Jabbari, R. Troncoso, and C. Zwikel, Spacetime Structure Near Generic Horizons and Soft Hair, Phys. Rev. Lett. 124, 041601 (2020).

[33] R. Haag, J. T. Lopuszanski, and M. Sohnius, All possible generators of supersymmetries of the s matrix, Nucl. Phys. B88, 257 (1975).

[34] M. A. Awada, G. W. Gibbons, and W. T. Shaw, Conformal supergravity, twistors and the super BMS group, Ann. Phys. (N.Y.) 171, 52 (1986).

[35] M. Henneaux, J. Matulich, and T. Neogi, Asymptotic realization of the super-BMS algebra at spatial infinity, Phys. Rev. D 101, 126016 (2020).

[36] S. G. Avery and B. U. W. Schwab, Residual Local Supersymmetry and the Soft Gravitino, Phys. Rev. Lett. 116, 171601 (2016).

[37] A. Fotopoulos, S. Stieberger, T. R. Taylor, and B. Zhu, Extended super BMS algebra of celestial CFT, J. High Energy Phys. 09 (2020) 198.

[38] O. Fuentealba, M. Henneaux, S. Majumdar, J. Matulich, and T. Neogi, Asymptotic structure of the Rarita-Schwinger theory in four spacetime dimensions at spatial infinity, arXiv:2011.04669.

[39] S. J. Haco, S. W. Hawking, M. J. Perry, and J. L. Bourjaily, The conformal BMS group, J. High Energy Phys. 11 (2017) 012.

[40] W. Nahm, Supersymmetries and their representations, Nucl. Phys. B135, 149 (1978).

[41] A. Ashtekar, J. Bicak, and B. G. Schmidt, Asymptotic structure of symmetry reduced general relativity, Phys. Rev. D 55, 669 (1997).

[42] G. Barnich and G. Compere, Classical central extension for asymptotic symmetries at null infinity in three spacetime dimensions, Classical Quantum Gravity 24, F15 (2007).

[43] G. Barnich, L. Donnay, J. Matulich, and R. Troncoso, Asymptotic symmetries and dynamics of three-dimensional flat supergravity, J. High Energy Phys. 08 (2014) 071.

[44] H. Adami, M. M. Sheikh-Jabbari, V. Taghiloo, H. Yavartanoo, and C. Zwikel, Symmetries at null boundaries: Two and three dimensional gravity cases, J. High Energy Phys. 10 (2020) 107.

[45] L. Donnay, G. Giribet, and F. Rosso, Quantum BMS transformations in conformally flat space-times and holography, J. High Energy Phys. 12 (2020) 102.

[46] C. Batlle, V. Campello, and J. Gomis, A canonical realization of the Weyl BMS symmetry, Phys. Lett. B 811, 135920 (2020).

[47] D. B. Fuks, Cohomology of Infinite-Dimensional Lie Algebras (Springer Science and Business Media, New York, 2012). 
[48] The $\operatorname{sl}(2, \mathbb{R})$ algebra is principally embedded within so $(3,2)$, if for any irreducible representation of the latter, the generators of the $\operatorname{sl}(2, \mathbb{R})$ subalgebra are irreducible. Note that in our case, since $\left\{\mathcal{J}_{m}, \mathcal{D}_{0}\right\}=0(m= \pm 1,0)$, the $\operatorname{sl}(2, \mathbb{R})$ subalgebra is reducible as it can be clearly seen in the adjoint representation, and thus, $\operatorname{sl}(2, \mathbb{R})$ turns out to be non-principally embedded within $s o(3,2)$.

[49] P. Bouwknegt and K. Schoutens, W symmetry, Adv. Ser. Math. Phys. 22, 1 (1995).

[50] L. Frappat, E. Ragoucy, and P. Sorba, W algebras and superalgebras from constrained WZW models: A group theoretical classification, Commun. Math. Phys. 157, 499 (1993).

[51] An isomorphism between the pure $\mathrm{BMS}_{3}$ algebra and the so-called W(2,2) algebra has also been pointed out in [87], and further elaborated in [88-93].

[52] Indeed, this sort of Sugawara-like construction can be successfully implemented in order to obtain the (super) $\mathrm{BMS}_{3}$ algebra from the affine extension of (super) Poincaré in 3D [94,95] (see also [96]).

[53] O. Coussaert, M. Henneaux, and P. van Driel, The asymptotic dynamics of three-dimensional Einstein gravity with a negative cosmological constant, Classical Quantum Gravity 12, 2961 (1995).

[54] J. H. Horne and E. Witten, Conformal Gravity in Threedimensions as a Gauge Theory, Phys. Rev. Lett. 62, 501 (1989).

[55] S. Deser, R. Jackiw, and S. Templeton, Topologically massive gauge theories, Ann. Phys. (N.Y.) 140, 372 (1982); , Erratum, Ann. Phys. (N.Y.) 185, 406 (1988).

[56] S. Deser, R. Jackiw, and S. Templeton, Three-Dimensional Massive Gauge Theories, Phys. Rev. Lett. 48, 975 (1982).

[57] J. Oliva, D. Tempo, and R. Troncoso, Static spherically symmetric solutions for conformal gravity in three dimensions, Int. J. Mod. Phys. A 24, 1588 (2009).

[58] J. Oliva, D. Tempo, and R. Troncoso, Three-dimensional black holes, gravitational solitons, kinks and wormholes for BHT massive gravity, J. High Energy Phys. 07 (2009) 011.

[59] H. Afshar, B. Cvetkovic, S. Ertl, D. Grumiller, and N. Johansson, Conformal Chern-Simons holography-lock, stock and barrel, Phys. Rev. D 85, 064033 (2012).

[60] M. Bertin, S. Ertl, H. Ghorbani, D. Grumiller, N. Johansson, and D. Vassilevich, Lobachevsky holography in conformal Chern-Simons gravity, J. High Energy Phys. 06 (2013) 015.

[61] H. R. Afshar, Flat/AdS boundary conditions in three dimensional conformal gravity, J. High Energy Phys. 10 (2013) 027.

[62] Here we mean highest weight in the Cartan decomposition of $s o(3,2)$. Highest weight generators then correspond to the set that commutes with $\mathcal{J}_{-1}$, given by $\left(\mathcal{J}_{-1}, \mathcal{P}_{-1}, \mathcal{K}_{-1}\right.$, $\left.\mathcal{D}_{0}\right)$.

[63] M. Henneaux, A. Perez, D. Tempo, and R. Troncoso, Chemical potentials in three-dimensional higher spin antide Sitter gravity, J. High Energy Phys. 12 (2013) 048.

[64] C. Bunster, M. Henneaux, A. Perez, D. Tempo, and R. Troncoso, Generalized black holes in three-dimensional spacetime, J. High Energy Phys. 05 (2014) 031.

[65] T. Regge and C. Teitelboim, Role of surface integrals in the Hamiltonian formulation of general relativity, Ann. Phys. (N.Y.) 88, 286 (1974).
[66] G. Barnich and F. Brandt, Covariant theory of asymptotic symmetries, conservation laws and central charges, Nucl. Phys. B633, 3 (2002).

[67] O. Aharony, O. Bergman, D. L. Jafferis, and J. Maldacena, $N=6$ superconformal Chern-Simons-matter theories, M2branes and their gravity duals, J. High Energy Phys. 10 (2008) 091.

[68] P. van Nieuwenhuizen, Three-dimensional conformal supergravity and Chern-Simons terms, Phys. Rev. D 32, 872 (1985).

[69] M. Rocek and P. van Nieuwenhuizen, $N>=2$ supersymmetric Chern-Simons terms as $d=3$ extended conformal supergravity, Classical Quantum Gravity 3, 43 (1986).

[70] A. Bagchi, S. Detournay, and D. Grumiller, Flat-Space Chiral Gravity, Phys. Rev. Lett. 109, 151301 (2012).

[71] A. Bagchi, R. Basu, S. Detournay, and P. Parekh, Flatspace chiral supergravity, Phys. Rev. D 97, 106020 (2018).

[72] N. Banerjee, D. P. Jatkar, I. Lodato, S. Mukhi, and T. Neogi, Extended supersymmetric $\mathrm{BMS}_{3}$ algebras and their free field realisations, J. High Energy Phys. 11 (2016) 059.

[73] I. Lodato and W. Merbis, Super-BMS 3 algebras from $\mathcal{N}=$ 2 flat supergravities, J. High Energy Phys. 11 (2016) 150.

[74] N. Banerjee, I. Lodato, and T. Neogi, $N=4$ supersymmetric BMS3 algebras from asymptotic symmetry analysis, Phys. Rev. D 96, 066029 (2017).

[75] R. Basu, S. Detournay, and M. Riegler, Spectral flow in 3D flat spacetimes, J. High Energy Phys. 12 (2017) 134.

[76] O. Fuentealba, J. Matulich, and R. Troncoso, Asymptotic structure of $\mathcal{N}=2$ supergravity in 3D: Extended super$\mathrm{BMS}_{3}$ and nonlinear energy bounds, J. High Energy Phys. 09 (2017) 030.

[77] A. Bagchi, A. Banerjee, S. Chakrabortty, and P. Parekh, Inhomogeneous tensionless superstrings, J. High Energy Phys. 02 (2018) 065.

[78] R. R. Poojary and N. V. Suryanarayana, On asymptotic symmetries of $3 \mathrm{~d}$ extended supergravities, J. High Energy Phys. 02 (2019) 168.

[79] H. Afshar, A. Bagchi, R. Fareghbal, D. Grumiller, and J. Rosseel, Spin-3 Gravity in Three-Dimensional Flat Space, Phys. Rev. Lett. 111, 121603 (2013).

[80] H. A. Gonzalez, J. Matulich, M. Pino, and R. Troncoso, Asymptotically flat spacetimes in three-dimensional higher spin gravity, J. High Energy Phys. 09 (2013) 016.

[81] M. Gary, D. Grumiller, M. Riegler, and J. Rosseel, Flat space (higher spin) gravity with chemical potentials, J. High Energy Phys. 01 (2015) 152.

[82] J. Matulich, A. Perez, D. Tempo, and R. Troncoso, Higher spin extension of cosmological spacetimes in 3D: Asymptotically flat behaviour with chemical potentials and thermodynamics, J. High Energy Phys. 05 (2015) 025.

[83] O. Fuentealba, J. Matulich, and R. Troncoso, Extension of the Poincaré group with half-integer spin generators: Hypergravity and beyond, J. High Energy Phys. 09 (2015) 003.

[84] O. Fuentealba, J. Matulich, and R. Troncoso, Asymptotically flat structure of hypergravity in three spacetime dimensions, J. High Energy Phys. 10 (2015) 009.

[85] R. Caroca, P. Concha, E. Rodríguez, and P. SalgadoRebolledo, Generalizing the $\mathfrak{b m s}_{3}$ and 2D-conformal algebras by expanding the Virasoro algebra, Eur. Phys. J. C 78, 262 (2018). 
[86] R. Caroca, P. Concha, O. Fierro, and E. Rodríguez, On the supersymmetric extension of asymptotic symmetries in three spacetime dimensions, Eur. Phys. J. C 80, 29 (2020).

[87] J. Rasmussen and C. Raymond, Galilean contractions of $W$ algebras, Nucl. Phys. B922, 435 (2017).

[88] N. Aizawa and Y. Kimura, Galilean conformal algebras in two spatial dimension, arXiv:1112.0634.

[89] D. Adamovic and G. Radobolja, Self-dual and logarithmic representations of the twisted Heisenberg-Virasoro algebra at level zero, Commun. Contemp. Math. 21, 1850008 (2019).

[90] W. Zhang and C. Dong, W-algebra W(2,2) and the vertex operator algebra $\mathrm{L}(1 / 2,0) \otimes \mathrm{L}(1 / 2,0)$, arXiv:0711.4624.

[91] W. Jiang and W. Zhang, Verma modules over the $\mathrm{W}(2,2)$ algebras, J. Geom. Phys. 98, 118 (2015).
[92] D. Adamovic and G. Radobolja, On free field realizations of W(2,2)-modules, SIGMA 12, 113 (2016).

[93] T. Araujo, Remarks on BMS3 invariant field theories: Correlation functions and nonunitary CFTs, Phys. Rev. D 98, 026014 (2018).

[94] G. Barnich and H. A. Gonzalez, Dual dynamics of three dimensional asymptotically flat Einstein gravity at null infinity, J. High Energy Phys. 05 (2013) 016.

[95] G. Barnich, L. Donnay, J. Matulich, and R. Troncoso, Super-BMS $\mathrm{B}_{3}$ invariant boundary theory from threedimensional flat supergravity, J. High Energy Phys. 01 (2017) 029.

[96] N. Banerjee, A. Bhattacharjee, Neetu, and T. Neogi, New $\mathcal{N}=2$ SuperBMS $_{3}$ algebra and invariant dual theory for 3D supergravity, J. High Energy Phys. 11 (2019) 122. 